\title{
Vasomotor symptoms in midlife women with incident breast cancer: pink SWAN
}

\author{
Ellen B. Gold ${ }^{1,7}\left(\mathbb{1} \cdot\right.$ Sybil L. Crawford ${ }^{2}\left([) \cdot\right.$ Katherine Leung $^{2}\left(\mathbb{0} \cdot\right.$ Gail Greendale $^{3} \cdot$ Katherine W. Reeves $^{4}(1) \cdot$ \\ Hadine Joffe ${ }^{5} \cdot$ Nancy E. Avis ${ }^{6}$
}

Received: 29 April 2021 / Accepted: 14 October 2021 / Published online: 25 October 2021

(c) The Author(s) 2021

\begin{abstract}
Purpose We compared trajectories of vasomotor symptoms (VMS) and their risk factors in women with breast cancer (BrCa) to those of cancer-free controls.

Methods Data were from 15 nearly annual follow-up visits (1996-2017) of the multi-racial/ethnic cohort of midlife women enrolled in the Study of Women's Health Across the Nation (SWAN). We compared women with incident BrCa to controls for patterns of VMS, controlling for risk factors identified in bivariate analyses using multivariable longitudinal analyses.

Results Characteristics at study entry largely did not differ between cases $(n=151)$ and controls $(n=2161)$. Adjusted prevalence of any VMS increased significantly among cases from diagnosis to 2.75 years post diagnosis [per-year adjusted odds ratio $(\mathrm{aOR})=1.76,95 \%$ confidence interval $(\mathrm{CI}) 1.39-2.24]$, peaking at 2.75 years post diagnosis, whereas prevalence was stable among controls in this interval $[\mathrm{aOR}=1.04,95 \% \mathrm{CI} 0.99-1.11]$. Beyond 2.75 years post diagnosis, prevalence declined significantly in cases [aOR $=0.72,95 \% \mathrm{CI} 0.61-0.84$ ] and less in controls [aOR $=0.96,95 \% \mathrm{CI} 0.92-1.00]$. Patterns were similar for frequent VMS. Adjustment for tamoxifen use slightly reduced the per-year OR for any prevalent VMS post diagnosis, partially explaining excess VMS in cases. Other treatments were unassociated with VMS.

Conclusions Patterns of prevalent VMS reporting differed significantly between cases and controls, particularly post diagnosis, the latter only partially explained by tamoxifen use among cases. Risk factors for VMS largely did not differ between cases and controls.
\end{abstract}

Keywords Breast cancer $\cdot$ Vasomotor symptoms $\cdot$ Risk factors $\cdot$ Menopause

Ellen B. Gold

ebgold@ucdavis.edu

1 University of California Davis School of Medicine, Davis, USA

2 University of Massachusetts Medical School, Worcester, USA

3 David Geffen School of Medicine, University of California Los Angeles, Los Angeles, USA

4 University of Massachusetts Amherst School of Public Health and Health Sciences, Amherst, USA

5 Harvard Medical School, Brigham and Women's Hospital, Boston, USA

6 Wake Forest University School of Medicine, Winston-Salem, USA

7 Department of Public Health Sciences, University of California Davis, One Shields Ave., Med Sci 1C, Davis, CA 95616, USA

\section{Introduction}

Breast cancer $(\mathrm{BrCa})$ is the most frequently occurring cancer among US women. Currently, about 3.8 million $\mathrm{BrCa}$ survivors are alive in the US (https://www.bcrf.org/breastcancer-statistics-and-resources), and $89.9 \%$ of the estimated 268,600 women diagnosed with $\mathrm{BrCa}$ annually will be alive 5 years after diagnosis (https://seer.cancer.gov/statfacts/ $\mathrm{html} / \mathrm{breast}$.html), a number projected to increase. Vasomotor symptoms (VMS) are the most frequent symptoms for women undergoing the menopausal transition (MT) [1, 2]. Greater prevalence [3], frequency (6 vs. 3.1 per $24 \mathrm{~h}$ [4]), and severely troubling VMS (quite or extremely bothered $55.4 \%$ vs. $12.1 \%$ [5]) have been reported by a greater proportion of women treated for $\mathrm{BrCa}$ than among those without a BrCa history, particularly among those taking aromatase inhibitors (AIs) [6, 7] or tamoxifen [8] or experiencing treatment-induced menopause $[9,10]$. VMS can adversely affect 
sleep [9], quality of life [10,11], depressive symptoms [12] and adherence to $\mathrm{BrCa}$ treatment [13-15], which may affect survival [16-18], although those with AI-associated VMS have improved disease-free survival (adjusted hazard ratio [aHR] 0.47) [19].

The Australian Longitudinal Study on Women's Health showed no association of VMS with incident $\mathrm{BrCa}$ (aHR 1.09) [20]. In the Life and Longevity After Cancer study, post-diagnosis VMS were significantly associated with chemotherapy (aOR 1.80), adjuvant hormone therapy (aOR 2.73), prior VMS (aOR 2.20), and older age [21]. The Study of Women's Health Across the Nation (SWAN) found VMS were protective for $\mathrm{BrCa}$ incidence (aHR 0.63) during 11.4 years of follow-up [22]. Here, we extended follow-up to 15 SWAN visits and investigated VMS pre- and post-diagnosis to elucidate whether VMS patterns differed between women with and without $\mathrm{BrCa}$ or reflected $\mathrm{BrCa}$ treatment effects. Additionally, we compared risk factors for VMS in women with $\mathrm{BrCa}$ to those without cancer. We addressed the following hypotheses:

(1) among women diagnosed with $\mathrm{BrCa}$ during followup, incidence and prevalence rates of VMS would be higher after diagnosis and cancer treatment than before;

(2) prevalence rates of VMS in $\mathrm{BrCa}$ cases would be lower than in controls pre-diagnosis but higher post diagnosis;

(3) in cases, treatment with BrCa-related selective estrogen receptor modulators (BrCa SERMS), endocrine medications, chemotherapy, or radiotherapy would be associated with greater prevalent VMS post treatment; and.

(4) risk factors for VMS pre- and post-diagnosis among $\mathrm{BrCa}$ cases would not differ from controls and those found previously in SWAN [2].

\section{Methods}

\section{Study participants}

SWAN is a seven-site, longitudinal study of a multi-racial/ ethnic cohort, characterizing physiological and psychosocial changes during the MT and assessing their relations to subsequent health. Each site recruited community-dwelling midlife women using random-digit dialing and/or list-based sampling [23] to identify Caucasians and one minority sample (African Americans in Boston, Pittsburgh, Chicago and Detroit; Hispanics in Newark, New Jersey; Japanese in Los Angeles; and Chinese in Oakland, CA). SWAN's crosssectional survey screened women for eligibility for the longitudinal study. Eligibility for the cross-sectional study included: resident in the geographic area of one of the sites, age 40-55 years, one of the target racial/ethnic groups, and spoke English or Japanese in Los Angeles, Spanish in New Jersey, or Cantonese in Oakland. Cohort eligibility included: aged 42-52 years, at least one menstrual period in the prior three months, not pregnant or lactating and not using exogenous sex steroids. Of 16,065 cross-sectional participants, 6521 were cohort eligible, and 3302 (50.6\%) enrolled.

For this study (Pink SWAN), cases were women who developed BrCa since SWAN's enrollment and had no cancer (except non-melanoma skin cancer) prior to developing $\mathrm{BrCa}$. Controls comprised women with no cancer (other than non-melanoma skin cancer) prior to their index visit (defined below). Data for cases were censored after any recurrence or other cancer that developed after their $\mathrm{BrCa}$ diagnosis and for controls after any cancer developed after their index visit. Over 15 follow-up visits, we identified 151 incident $\mathrm{BrCa}$ cases and 2161 controls (Fig. 1). BrCa risk status was not ascertained for controls.

Adjudication of participant medical records began at visit 12. Of 109 cases for whom medical records were received, 103 cases were confirmed $(94.5 \%$ agreement with selfreport). For each case, we ascertained the date of diagnosis. To assign a corresponding date in controls, an index date was randomly assigned, using frequency matching to cases for first post-diagnosis visit, and randomly assigning a date between the last pre-diagnosis visit and the first post-diagnosis visit, such that the distributions of diagnosis/index dates were comparable in cases and controls.

Exclusions were: $\mathrm{BrCa}$ diagnosed prior to baseline $(N=28)$; other cancer diagnosed prior to baseline $(N=37)$ or between baseline and diagnosis/index visit $(N=104)$; and potential controls who missed a study visit corresponding to their randomly assigned index date $(N=885)$ (Fig. 1$)$. Of these 885,716 did not have at least one visit prior to and one visit after the index visit, so that interpolating data for a visit was not feasible. Compared with included controls, those excluded were more likely to be from the New Jersey site and Hispanic, reflecting a hiatus in data collection for visits $6-8$ and $10-11$ at that site. The higher proportion of Hispanics likely explains the slightly younger age, higher body mass index (BMI), lower educational attainment, higher anxiety and depressive symptom scores, and greater proportion of smokers among those excluded. Multivariate analyses adjusted for these characteristics.

The study protocol common to all sites was approved by each site's institutional review board, and all participants provided written, signed informed consent. 


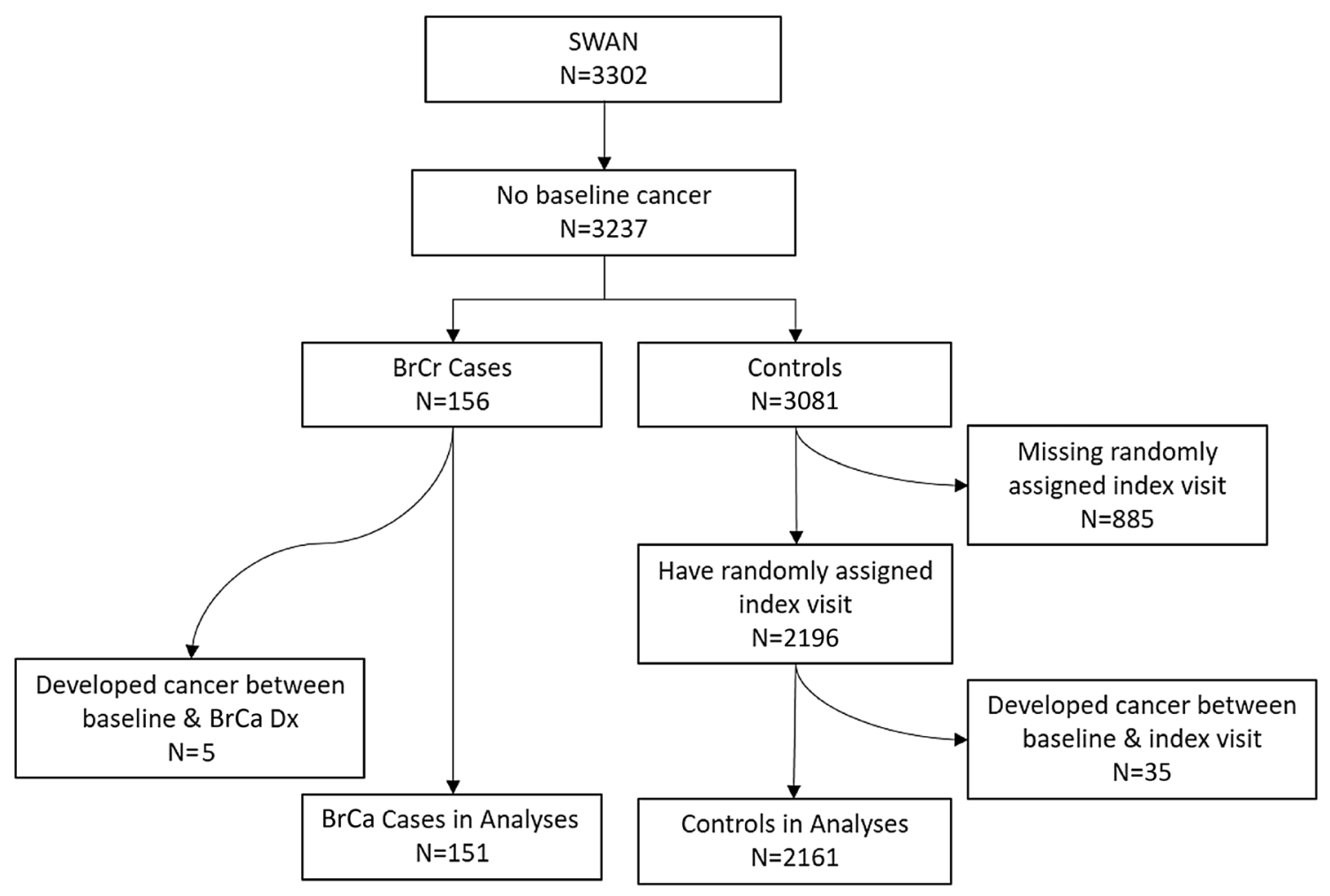

Fig. 1 Flowchart for derivation of case and control samples

\section{Data collection}

\section{Procedures}

Participants were followed with approximately annual inperson visits from 1996 to 2017. All visits included: consent; a standard protocol for measuring height with a stadiometer and weight with a balance beam scale; and interviewer- and self-administered questionnaires, including questions on demographic characteristics, medical and reproductive history, medications, psychosocial measures, and symptoms. Questionnaires were forward- and back-translated into Spanish, Cantonese, and Japanese and administered by bilingual interviewers to participants preferring these languages.

\section{Outcome variables}

At each visit, participants were asked the frequency of their hot flashes and/or night sweats in the prior 2 weeks [24-26]. Prior analyses [2] indicated that these two symptoms were highly correlated, providing justification for examining them jointly. Any VMS was defined as reporting at least 1 day with hot flashes or night sweats within the prior 2 weeks, and frequent VMS as having VMS on 6 or more days in the prior 2 weeks [2].

\section{Covariates}

Time-invariant factors included race/ethnicity, site, visit 1 symptom sensitivity [27], menopausal stage and use of systemic menopausal hormone therapy (HT) at the index visit, baseline history of premenstrual symptoms, education, and ever receiving $\mathrm{BrCa}$ chemotherapy or radiotherapy (treatment timing was unavailable). Chemotherapy and radiotherapy were ascertained from interviews, medical records, and self-reported questionnaires from cases.

All other covariates were time-varying. Because current smoking [28] was stable, missing values were interpolated using last observation carried forward. Passive smoke exposure [29] and alcohol consumption were missing in more than 2700 observations and thus excluded; adjustment for these two characteristics yielded similar results in the subsample for whom they were available (data not shown). Psychosocial characteristics included elevated anxiety score [24] for the prior two weeks (score $>4$ [30]) and elevated depressive symptom score (score $\geq 16$ on the 20 -item Center for Epidemiology Studies-Depression (CES-D) scale for the previous week [31]), both lagged one visit. Marital status was categorized as never, previously, or currently married/ partnered. Self-assessed health was categorized as excellent, very good, good, and fair/poor. BMI was computed 
as weight in $\mathrm{kg} /(\text { height in meters })^{2}$, categorized as $<25$, 25-29.9, 30+.

At all visits except 11 and 14, interviewers ascertained prescription medication use since the prior visit, transcribing preparation names from medication containers that participants brought into the visit. Medications analyzed here included HT, anti-anxiety medications, anti-depressants, BrCa SERMS (tamoxifen for all but one case), non-BrCa SERMs, and endocrine medications. BrCa treatment information (BrCa SERMS, endocrine medications [including aromatase inhibitors, GnRH agonists and (less frequently reported in our participants) estrogen receptor blockers and inhibitors of Type II $5 \alpha$-reductase], chemotherapy and radiotherapy) was obtained in annual surveys and from a Pink SWAN questionnaire and medical records for women who reported incident $\mathrm{BrCa}$. Medications were coded using the Iowa Drug Information Service system. Follow-up visits 11 and 14 were abbreviated; medications other than HT, employment, and self-reported health were not ascertained. Values were imputed using logistic or linear regression, based on adjacent visit values, age, menopausal status, race/ ethnicity, site, and case/control status.

Menopausal status categories-both at the index visit and time-varying-included premenopausal (menses in the prior three months without change in menstrual regularity in the prior year), early peri-menopausal (menses in the prior 3 months and changes in regularity in the prior year), late peri-menopausal (3-11 months of amenorrhea) and naturally postmenopausal ( $\geq 12$ months of amenorrhea). Women who had a hysterectomy and/or bilateral oophorectomy were grouped with naturally postmenopausal women at the index visit due to small numbers of such surgeries and similar rates of VMS in the two groups but were separated for timevarying menopausal status. Pre- and early peri-menopausal stages were combined due to the small number of premenopausal observations post diagnosis.

For women initiating menopausal HT or oral contraception use before their final menstrual period, menopausal status could not be discerned because menstrual bleeding was masked. If a woman stopped such hormone use and resumed bleeding, menopausal status was categorized according to the criteria above. Those who had the same status at both the visits prior to and following hormone use were categorized with that menopausal stage; otherwise menopausal status was considered missing.

\section{Data analyses}

Participant characteristics were compared for cases and controls using chi-square or Fisher's exact test for categorical variables and Student's $t$ test for continuous variables.

In addition to case/control status, the primary predictor was years before/after diagnosis/index date with diagnosis/ index date $=$ time 0 . Diagnosis date was used as a surrogate for treatment initiation, which we assumed occurred shortly after diagnosis. Analyses of prevalent VMS omitted data outside the range -10 to +10 years to avoid sparse data.

Patterns of prevalent and incident VMS were examined in relation to the diagnosis/index date to distinguish patterns of persistent VMS in women who already had VMS from newly developed VMS, respectively. For both prevalence and incidence, any and frequent VMS were modeled separately.

Prevalence of VMS was modeled using random effects logistic regression [32]. Separately for cases and controls, we used nonparametric, locally weighted scatterplot smoothing (LOESS) regression by years before/after diagnosis/index [33]. Possible knots-points of inflection with a change in slope-suggested by LOESS were tested in piecewise linear modeling to identify the best-fitting knot placement. To allow cases and controls to have different slopes in segments delineated by knots, we included interactions of case/control status and segment-specific time variables in logistic regression models. Results from these models are presented as: (a) point prevalences at segment start and (b) segmentspecific per-year adjusted odds ratios (aOR); the former indicates the point prevalence level at the start of segments, and the latter reflects change in odds with an additional 1 year elapsed.

To address hypothesis \#1, in cases we compared point prevalence (PP) of VMS at diagnosis and post-diagnosis, and per-year aORs in different segments. For hypothesis \#2, we compared PP and per-year aORs for cases versus controls in each segment. Covariate adjustment was performed in three stages: covariates other than $\mathrm{BrCa}$ treatments; adding medications (hypothesis \#3); and adding chemotherapy and radiotherapy (hypothesis \#3). For hypothesis \#4, we tested interactions between case/control status and VMS risk factors previously identified in SWAN [2], stratifying on pre and post diagnosis/index. We also examined changes in HT use, as cases may have been more likely to stop HT postdiagnosis, potentially leading to "rebound" VMS.

Incidence of VMS was analyzed in participants without baseline VMS using Kaplan-Meier plots-and covariateadjusted discrete survival analysis accounting for "late entry"-participants were excluded from risk sets earlier than their study entry [34].

All analyses used SAS 9.4 (SAS Institute Inc., Cary, NC, USA). $p$ values $<0.05$ were considered statistically significant. 
Table 1 Comparison of baseline characteristics of cases and controls

\begin{tabular}{|c|c|c|c|}
\hline & Controls $(N=2161)$ & $\begin{array}{l}\text { Breast Cancer } \\
\text { Cases }(N=151)\end{array}$ & $p$ value $^{\mathrm{a}}$ \\
\hline Race/ethnicity: $N(\%)$ & & & 0.54 \\
\hline White & $1045(48.4)$ & $75(49.7)$ & \\
\hline Black & $598(27.7)$ & $44(29.1)$ & \\
\hline Chinese & $196(9.1)$ & $10(6.6)$ & \\
\hline Japanese & $216(10.0)$ & $18(11.9)$ & \\
\hline Hispanic & $106(4.9)$ & $4(2.6)$ & \\
\hline Age in years: median, $\mathrm{IQR}^{\mathrm{b}}$ & $46.3(4.1)$ & $46.6(4.7)$ & 0.26 \\
\hline Educational level: $N(\%)$ & & & 0.36 \\
\hline No more than high school & $457(21.4)$ & $27(18.1)$ & \\
\hline Some college & $694(32.3)$ & $44(29.5)$ & \\
\hline College degree & $996(46.4)$ & $78(52.4)$ & \\
\hline Marital status: $N(\%)$ & & & 0.36 \\
\hline Currently married/living as married & $1470(68.8)$ & $94(63.5)$ & \\
\hline Never married & $263(12.3)$ & $23(15.5)$ & \\
\hline Previously married & $403(18.9)$ & $31(22.0)$ & \\
\hline Difficulty paying for basics: $N(\%)$ & & & 0.85 \\
\hline Not at all & $143(6.8)$ & $10(6.8)$ & \\
\hline Somewhat & $603(28.5)$ & $39(26.4)$ & \\
\hline Very & $1372(64.8)$ & $99(66.9)$ & \\
\hline Current employment status: $N(\%)$ & & & 0.93 \\
\hline No & $393(18.2)$ & $27(17.9)$ & \\
\hline Yes & $1768(81.8)$ & $124(82.1)$ & \\
\hline Self-reported health: $N(\%)$ & & & 0.44 \\
\hline Excellent & $493(23.1)$ & $34(23.0)$ & \\
\hline Very good & $806(37.7)$ & $50(33.8)$ & \\
\hline Good & $597(27.9)$ & $50(33.8)$ & \\
\hline Fair/poor & $242(11.3)$ & $14(9.5)$ & \\
\hline Smoking: $N(\%)$ & & & 0.44 \\
\hline Never & $1281(59.3)$ & $91(60.7)$ & \\
\hline Past & $577(26.7)$ & $34(22.7)$ & \\
\hline Current & $302(14.0)$ & $25(16.7)$ & \\
\hline Passive smoking exposure: $N(\%)$ & & & 0.88 \\
\hline None & $1003(46.7)$ & $67(44.7)$ & \\
\hline $1-4$ person-h per week & $587(27.3)$ & $42(28.0)$ & \\
\hline $5+$ person-h per week & $558(266.0)$ & $41(27.3)$ & \\
\hline Body mass index, $\mathrm{kg} / \mathrm{m}^{2}$ : median, IQR & $26.16(8.9)$ & $26.9(9.6)$ & 0.39 \\
\hline Alcohol use: $N(\%)$ & & & 0.029 \\
\hline None & $1022(49.6)$ & $61(41.5)$ & \\
\hline Light $(<1$ drink/week $)$ & $201(9.8)$ & $19(12.9)$ & \\
\hline Moderate (1-7 drinks/week) & $529(25.7)$ & $51(34.7)$ & \\
\hline Heavy (> 7 drinks/week) & $309(15.0)$ & $16(11.0)$ & \\
\hline CES-D score $\geq 16: N(\%)$ & & & 0.79 \\
\hline No & $1682(77.9)$ & $119(78.8)$ & \\
\hline Yes & $478(22.1)$ & $32(21.2)$ & \\
\hline Visit 01 symptom sensitivity: median (IQR) & $10.0(4.0)$ & $10.0(5.0)$ & 0.96 \\
\hline Anxiety ( $\geq 4$ on scale): $N(\%)$ & & & 0.76 \\
\hline No & $1894(87.8)$ & $133(88.7)$ & \\
\hline Yes & $263(12.2)$ & $17(11.3)$ & \\
\hline History of premenstrual symptoms: $(N) \%$ & & & 0.90 \\
\hline No & $241(11.2)$ & 17 (11.6) & \\
\hline
\end{tabular}


Table 1 (continued)

\begin{tabular}{lccc}
\hline & Controls $(N=2161)$ & $\begin{array}{l}\text { Breast Cancer } \\
\text { Cases }(N=151)\end{array}$ & $p$ value $^{\mathrm{a}}$ \\
\hline Yes & $1905(88.8)$ & $130(88.4)$ & 0.72 \\
Menopause transition stage: $N(\%)$ & $1177(54.5)$ & $80(53.0)$ & \\
Premenopausal & $984(45.5)$ & $71(47.0)$ & 0.37 \\
Early peri-menopausal & & $98(65.3)$ & \\
Vasomotor symptoms: $N(\%)$ & $1307(60.8)$ & $35(23.3)$ & \\
0 days in past 2 weeks & $616(28.7)$ & $17(11.3)$ & \\
1-5 days in past 2 weeks & $226(10.5)$ & & \\
6+ days in past 2 weeks &
\end{tabular}

${ }^{\mathrm{a}} \mathrm{Chi}$-square test for categorical variables, Kruskall-Wallis test for continuous variables

${ }^{\mathrm{b}}$ Interquartile range

\section{Results}

Controls had a slightly but not statistically significantly higher point prevalence (PP) of VMS at baseline (39.3\%) than cases (34.7\%) (Table 1). Demographic, psychosocial and health characteristics did not differ between cases and controls, except cases tended to drink more alcohol (Table 1). Factors associated with VMS at baseline largely did not differ from those previously reported in SWAN [2] (data not shown).

The LOESS plot of the PP of any VMS among cases (hypothesis \#1) suggested two knots with an increase starting at diagnosis (year 0) and a decrease starting at 2.75 years post-diagnosis (Fig. 2a). Among controls, VMS decreased gradually over time (hypothesis \#2). Patterns were similar for the PP for frequent VMS (Fig. 2b). Consequently, all models used two knots, estimating separate per-year aORs for cases and controls in each segment: Segment $1=$ up to 10 years pre-diagnosis/index; Segment $2=$ diagnosis/index to 2.75 years post-diagnosis/index; and Segment $3=2.75-10$ years post-diagnosis/index.

For hypothesis \#1, analyses only in cases, adjusted PP of any VMS declined slightly pre-diagnosis (per-year $\mathrm{aOR}=0.91$; Table 2, first model), but increased sharply in Segment 2 with PP at 2.75 years post diagnosis was almost twice the PP at diagnosis (77.9\% versus $42.6 \%, p<0.001)$. The per-year aOR for cases in Segment 2 is 1.76, indicating that for each additional passing year, the odds of any VMS increased by a factor of 1.76. Subsequently, prevalence rates declined (Segment 3 per-year aOR $=0.72$ ). Pairwise differences between segment-specific per-year aORs were statistically significant $(p<0.004)$.

In hypothesis \#2 analyses, adjusted PP of any VMS was somewhat higher in cases than controls pre-diagnosis/index $(p=0.053)$ (Table 2, first model), but decreased similarly for both groups in Segment $1(p=0.12)$. In Segment 2 , in contrast to the sharp increase in PP noted above in cases, $\mathrm{PP}$ in controls was stable (per-year aOR $=1.04$,
A

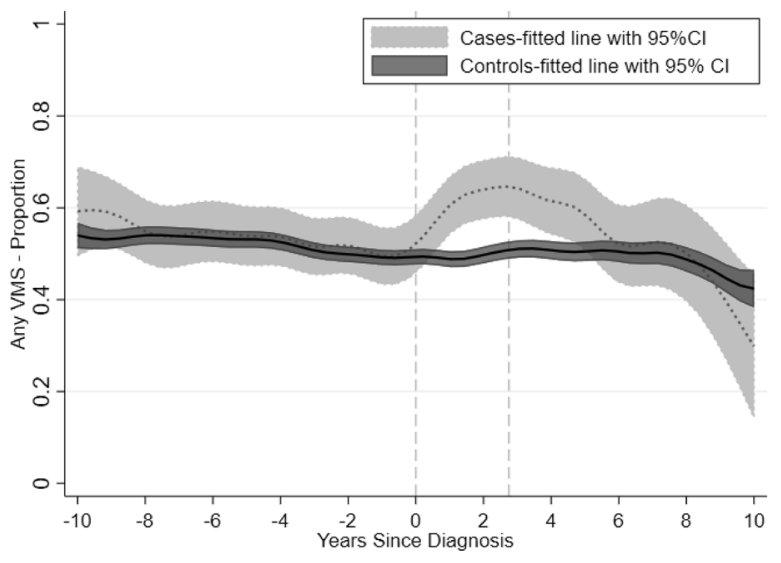

Vertical dashed lines indicate location of knots

B

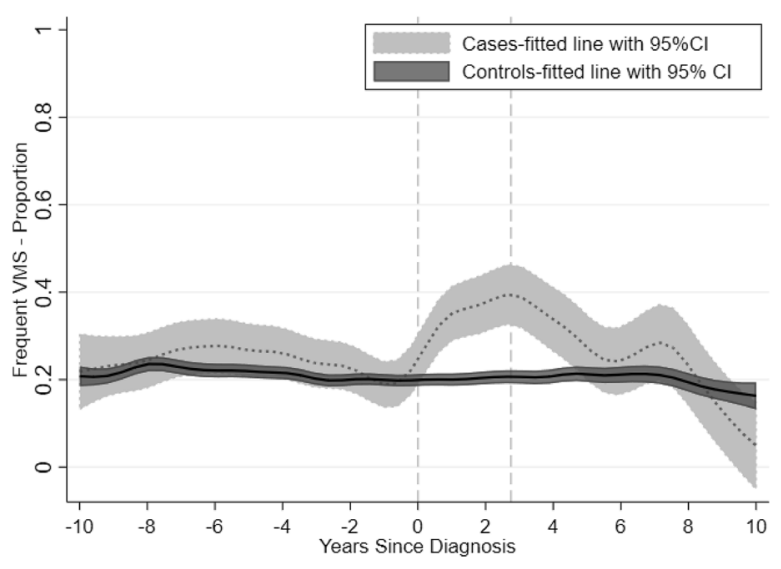

Vertical dashed lines indicate location of knots

Fig. 2 a Proportion any prevalent VMS, loess curve over time prior to and after diagnosis/index date for cases and controls. b Proportion Frequent Prevalent VMS, loess curve over time prior to and after diagnosis/index date for cases and controls

$p<0.001$ for case/control difference), leading to a 
Table 2 Multivariable random effects binomial logistic regression for prevalence of any VMS from 10 years pre- to 10 years post-diagnosis, Pink SWAN

\begin{tabular}{|c|c|c|c|c|c|c|}
\hline & \multicolumn{2}{|c|}{$\begin{array}{l}\text { Segment 1: -10 years to diagnosis/ } \\
\text { index }\end{array}$} & \multicolumn{2}{|c|}{$\begin{array}{l}\text { Segment } 2 \text { : diagnosis/index to } \\
2.75 \text { years post diagnosis/Index }\end{array}$} & \multicolumn{2}{|c|}{$\begin{array}{l}\text { Segment } 3: 2.75-10 \text { years post diagno- } \\
\text { sis/index }\end{array}$} \\
\hline & $\begin{array}{l}\text { Adjusted } \% \text { point } \\
\text { prevalence }(\mathrm{SE}) \text { at } \\
-10 \text { years }\end{array}$ & $\begin{array}{l}\text { Per-year odds } \\
\text { ratio }^{\text {a }}(95 \% \mathrm{CI})\end{array}$ & $\begin{array}{l}\text { Adjusted \% point } \\
\text { prevalence (SE) at } \\
\text { diagnosis/index }\end{array}$ & $\begin{array}{l}\text { Per-year odds } \\
\text { ratio }^{\text {a }}(95 \% \mathrm{CI})\end{array}$ & $\begin{array}{l}\text { Adjusted } \% \text { point } \\
\text { prevalence }(\mathrm{SE}) \\
\text { at }+2.75 \text { years }\end{array}$ & $\begin{array}{l}\text { Per-year odds } \\
\text { ratio }^{\text {a }}(95 \% \mathrm{CI})\end{array}$ \\
\hline \multicolumn{7}{|l|}{ Adjusted $^{\mathrm{b}}$} \\
\hline Controls & $51.0(3.3)$ & $0.97(0.94,0.99)$ & $42.3(2.2)$ & $1.04(0.99,1.11)$ & $45.2(2.7)$ & $0.96(0.92,1.00)$ \\
\hline Breast cancer cases & $65.4(7.2)$ & $0.91(0.84,0.98)$ & $42.6(6.2)$ & $1.76(1.39,2.24)$ & $77.9(5.4)$ & $0.72(0.61,0.84)$ \\
\hline $\begin{array}{l}p \text { Values, case-con- } \\
\text { trol difference }{ }^{\mathrm{c}}\end{array}$ & 0.053 & 0.123 & 0.964 & $<0.001$ & $<0.001$ & $<0.001$ \\
\hline \multicolumn{7}{|l|}{$\begin{array}{l}\text { Additional adjust- } \\
\text { ment for time- } \\
\text { varying non-BrCa } \\
\text { SERM, BrCa } \\
\text { SERM, and } \mathrm{BrCa} \\
\text { endocrine medica- } \\
\text { tions }\end{array}$} \\
\hline Controls & $51.1(3.3)$ & $0.97(0.94,0.99)$ & $42.4(2.2)$ & $1.04(0.98,1.11)$ & $45.2(2.7)$ & $0.96(0.92,1.00)$ \\
\hline Breast cancer cases & $65.3(7.2)$ & $0.91(0.84,0.98)$ & $41.7(6.2)$ & $1.56(1.20,2.04)$ & $71.0(7.5)$ & $0.77(0.65,0.91)$ \\
\hline $\begin{array}{l}p \text { Values, case-con- } \\
\text { trol difference }{ }^{\mathrm{c}}\end{array}$ & 0.057 & 0.107 & 0.916 & 0.0034 & 0.0026 & 0.0093 \\
\hline \multicolumn{7}{|l|}{$\begin{array}{l}\text { Further adjustment } \\
\text { for ever chemo- } \\
\text { and ever radio- } \\
\text { therapy }\end{array}$} \\
\hline Controls & $51.2(3.3)$ & $0.96(0.94,0.99)$ & $42.2(2.2)$ & $1.04(0.98,1.11)$ & $45.0(2.7)$ & $0.96(0.92,1.00)$ \\
\hline Breast cancer cases & $53.1(11.2)$ & $0.91(0.84,0.98)$ & $30.2(8.7)$ & $1.63(1.23,2.16)$ & $62.5(11.5)$ & $0.76(0.64,0.91)$ \\
\hline $\begin{array}{l}p \text { Values, case-con- } \\
\text { trol difference }\end{array}$ & 0.87 & 0.128 & 0.206 & 0.002 & 0.141 & 0.010 \\
\hline
\end{tabular}

a Per-year odds ratio reflects the change in odds associated with a 1-year increase in that segment

${ }^{\mathrm{b}}$ Adjusted for: menopause status and hormone use at first post-diagnosis visit, site, race/ethnicity and time-varying menopause transition stage and hormone use, age, body mass index, education, self-reported health, marital status, employment status, symptom sensitivity at visit 01 , 1-year lagged anxiety score $>4$, 1-year lagged anxiety medication use, and use of anti-depressants, history of premenstrual symptoms and smoking status

${ }^{c}$ For prevalences at start of segments, $p$ value indicates statistical significance for the case-control difference in VMS prevalence. For per-year ORs, the $p$ value indicates statistical significance for the case-control difference in the time slope, i.e., for the interaction between case-control status and segment time

significantly higher PP at 2.75 years post-diagnosis/index for cases than controls $(77.9 \%$ vs. $45.2 \%, p<0.001)$. Conversely, in Segment 3, VMS PP declined significantly in cases as noted above but remained stable in controls (per-year aOR $0.96 ; p<0.001$ for case/control difference). Adjusted PPs for frequent VMS were lower (6.7-9.5\% among controls, $7.6-27.1 \%$ among cases), but case/control differences in PP and per-year aORs were consistent with those for any VMS (data not shown).

New HT use at the first post-diagnosis/index visit was low in both cases and controls (3.9\% versus $4.3 \%$, respectively, $p=0.85$ ). However, continued use among cases was significantly lower than among controls at the first post-diagnosis visit $(28.6 \%$ versus $61.8 \%, p=0.003)$. Adjustment for HT patterns had a negligible effect on per-year aORs, however (data not shown), nor was the interaction of HT pattern with case/control status statistically significant $(p=0.67)$.

Use of BrCa SERMS occurred almost entirely among cases post-diagnosis $(29.0 \%$ of Segment 2 observations, $14.4 \%$ of Segment 3 observations), with only two Segment 1 observations $(0.01 \%)$ in controls. Endocrine medication use was reported in $15.6 \%$ and $18.2 \%$ of cases' Segment 2 and Segment 3 observations, respectively, versus $0.10 \%$ and $0.03 \%$ among controls. In hypothesis \#3 analyses, concurrent $\mathrm{BrCa}$ SERM use was positively associated with prevalence of any VMS (aOR $=2.95,95 \%$ CI 1.34, 6.52), but concurrent endocrine medication use was unrelated $(\mathrm{aOR}=0.96$, 95\% CI 0.46, 1.97). The latter result may reflect endocrine medication use only during postmenopause, rather than in late peri-menopause when VMS PP tends to peak [2]. 
Adjustment for medications reduced the Segment 2 per-year aOR for any VMS among cases from 1.76 to 1.56 (Table 2, second model), but it remained significantly higher than for controls $(p=0.003)$. The pattern was similar for prevalence of frequent VMS (data not shown).

Among cases, $41.3 \%$ received chemotherapy, and 70.9\% received radiotherapy. Neither was significantly associated with the prevalence of any VMS (chemotherapy aOR $=0.96$, $95 \%$ CI $0.43,2.15$; radiotherapy $\mathrm{aOR}=1.86,95 \%$ CI 0.79 , 4.37), nor did adjustment affect case/control differences in PPs of VMS and per-year aORs (Table 2, third model).

\section{A}

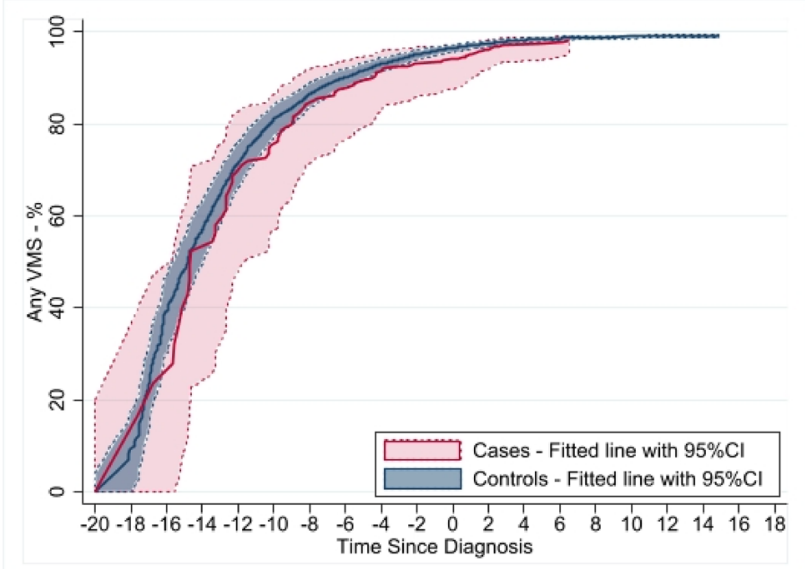

Unadjusted hazard ratio, case vs. control, $=0.87(0.70,1.08), p=0.207$

B

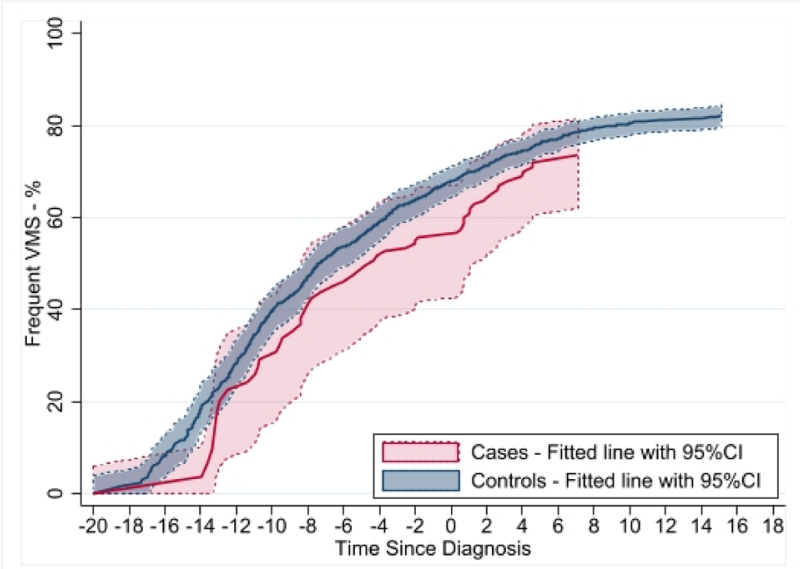

Unadjusted hazard ratio, case vs. control, $=0.86(0.66,1.13), \mathrm{p}=0.287$

Fig. 3 a Kaplan-Meier Plot of cumulative probability (with 95\% Confidence Intervals) of any incident VMS over time prior to and after diagnosis/index date for cases and controls. b Kaplan-Meier plot of cumulative probability (with 95\% Confidence Intervals) of frequent incident VMS over time prior to and after diagnosis/index date for cases and controls
Similar to previous SWAN findings [22], Kaplan-Meier plots suggested slightly lower incidence of any VMS (Fig. 3a) and frequent VMS (Figs. 3b) in cases than controls with almost all incidence for both groups occurring prediagnosis/index. This resulted in small numbers of incident VMS post-diagnosis/index (19-21 incidents in cases), making multivariate analyses of these trends unstable. Overall, aHR (95\% CI) for cases versus controls were $1.16(0.79$, $1.70)$ for any VMS and $1.10(0.74,1.63)$ for frequent $V M S$. Among participants with no VMS pre-diagnosis, corresponding aHRs post-diagnosis/index were $1.37(0.61,3.07)$ and $1.55(0.84,2.87)$, respectively.

In hypothesis \#4 analyses, no statistically significant case/control interactions with risk factors were observed for prevalence of frequent VMS, either pre- or post-diagnosis/ index. For prevalence of any VMS, two interactions were statistically significant: race/ethnicity pre-diagnosis (interaction $p=0.043$ ) and menopause status post-diagnosis (interaction $p=0.026$ ): aORs for Black versus white were 9.04 for cases versus 2.09 for controls, and for Hispanic versus white were 24.4 for cases versus 0.52 for controls; aORs for late versus early peri-menopause were 13.0 for cases but 3.77 for controls; aORs for post- versus early peri-menopause were 12.5 for cases but 2.80 for controls. Given the large number of comparisons and small cell counts (7 Hispanic observations, 33 late peri-menopause observations, and 36 pre-/early peri-menopause in cases), these significant interactions may have been due to chance.

\section{Discussion}

Our results provide a new contribution by comparing preand particularly post-diagnosis trajectories of VMS among women with incident $\mathrm{BrCa}$ to those of controls, revealing that much of the increase post-diagnosis was not attributable to treatment, and revealed that VMS risk factors did not differ in cases and controls.

Point prevalence of any VMS was similar for both groups pre-diagnosis/index, consistent with prior SWAN findings [20]. This differed from findings from a nearly 18-year follow-up of 25,499 postmenopausal non-HT users in the Women's Health Initiative, which revealed that women with VMS lasting $10+$ years had higher $\mathrm{BrCa}$ incidence than those who never reported VMS [35]. In our study, the PP of VMS increased in cases from diagnosis to +2.75 years but remained stable among controls, suggesting treatment effects in cases, supported by the significance of the BrCa SERMs variable, consistent with prior findings [21]. However, adjustment for BrCa SERM treatment only somewhat attenuated the still significantly elevated per-year aOR among cases, suggesting that treatment only partially accounted for the VMS increase in contrast to findings from prior studies 
of $\mathrm{BrCa}$ cases taking aromatase inhibitors $[6,7]$ or tamoxifen [8]. Consistent with prior results [3], chemotherapy had no additional impact on VMS prevalence, which declined in cases and controls after 2.75 years after diagnosis/index with a steeper decline among cases.

Most incident VMS in both groups occurred pre-diagnosis/index, resulting in small numbers of incident VMS post diagnosis/index; thus, incidence trends were unstable, but indicated no statistically significant case/control differences in post-diagnosis/index incident VMS. Because analyses of prevalent VMS included women who had VMS in prior segments, but analyses of incident VMS did not, the higher per-year aOR for prevalence of VMS among cases (after adjustment for BrCa SERMs), but not incidence of VMS in Segment 2 could reflect a predisposition for VMS among cases or common underlying factors (eg, inflammation) for $\mathrm{BrCa}$ [36] and VMS [37].

Our study's strengths included: (a) the longitudinal design, permitting assessment of temporal relations of VMS, BrCa and VMS risk factors; (b) inclusion of five racial/ethnic groups, providing good generalizability; and (c) standardized data, enabling statistical adjustment for many potential covariates, reducing residual confounding. The study had limitations, however. First, most notably, the number of incident $\mathrm{BrCa}$ cases was relatively small, potentially resulting in some modest but meaningful associations not detected as statistically significant, such as when examining incident VMS and case/control status interaction with risk factors. Second, recall errors and misclassification could have resulted from self-reported data on VMS, BrCa treatments, and medications; however, we verified reported medications against containers for prescribed medications that women brought to each visit. Third, we lacked information regarding timing of chemotherapy or radiotherapy so that we could not examine concurrent treatment but only any vs. no treatment, which may explain the lack of significant associations. Finally, most cases occurred postmenopausally so that the menopause-inducing effects, including VMS, of some $\mathrm{BrCa}$ therapies reported in prior studies $[9,10]$ were not observable.

In conclusion, risk factors for VMS did not differ between cases and controls, but trajectories of prevalence of VMS differed significantly. Prevalence of VMS remained stable among controls, but increased in cases in the immediate post-diagnosis segment, partially reflecting treatment but also possibly reflecting common underlying factors for $\mathrm{BrCa}$ and VMS or a predisposition for VMS among cases. However, incidence of VMS did not increase post- diagnosis, arguing against predisposition, but not against common factors for $\mathrm{VMS}$ and $\mathrm{BrCa}$. These findings suggest that prevalence of VMS post-diagnosis was only partially explained by treatment and possibly by prior prevalence of VMS in cases because analyses of VMS prevalence included women who had VMS previously, again suggesting shared underlying factors for $\mathrm{VMS}$ and $\mathrm{BrCa}$. That post-diagnosis VMS were not entirely attributable to treatments which is important clinically because VMS could lead to lower adherence to $\mathrm{BrCa}$ treatment. Additionally, we did not find higher HT use in cases than controls pre-diagnosis so that higher prevalence of VMS soon after BrCa diagnosis does not seem attributable to prior HT use.

Acknowledgements The Study of Women's Health Across the Nation (SWAN) has grant support from the National Institutes of Health (NIH), DHHS, through the National Institute on Aging (NIA), the National Institute of Nursing Research (NINR), and the NIH Office of Research on Women's Health (ORWH) (Grants U01NR004061; U01AG012505, U01AG012535, U01AG012531, U01AG012539, U01AG012546, U01AG012553, U01AG012554, U01AG012495). Pink SWAN has grant support from the National Cancer Institute (CA 199137). The content of this article is solely the responsibility of the authors and does not necessarily represent the official views of the NIA, NINR, ORWH, or the NIH. Clinical Centers: University of Michigan, Ann Arbor-Siobán Harlow, PI 2011—present, MaryFran Sowers, PI 1994-2011; Massachusetts General Hospital, Boston, MA-Joel Finkelstein, PI 1999_-present; Robert Neer, PI 1994-1999; Rush University, Rush University Medical Center, Chicago, IL-Howard Kravitz, PI 2009—present; Lynda Powell, PI 1994-2009; University of California, Davis/Kaiser-Ellen Gold, PI; University of California, Los Angeles-Gail Greendale, PI; Albert Einstein College of Medicine, Bronx, NY - Carol Derby, PI 2011 - present, Rachel Wildman, PI 2010 - 2011; Nanette Santoro, PI 2004-2010; University of Medicine and Dentistry-New Jersey Medical School, Newark-Gerson Weiss, PI 1994-2004; and the University of Pittsburgh, Pittsburgh, PA-Karen Matthews, PI. NIH Program Office: National Institute on Aging, Bethesda, MD-Chhanda Dutta 2016- present; Winifred Rossi 2012-2016; Sherry Sherman 1994-2012; Marcia Ory 1994-2001; National Institute of Nursing Research, Bethesda, MD-Program Officers. Central Laboratory: University of Michigan, Ann ArborDaniel McConnell (Central Ligand Assay Satellite Services). Coordinating Center: University of Pittsburgh, Pittsburgh, PA-Maria Mori Brooks, PI 2012—present; Kim Sutton-Tyrrell, PI 2001-2012; New England Research Institutes, Watertown, MA-Sonja McKinlay, PI 1995-2001. Steering Committee: Susan Johnson, Current Chair; Chris Gallagher, Former Chair. We thank the study staff at each site and all the women who participated in SWAN.

Author contributions EBG, SLC, GG, and NEA participated in the design of the study, data collection instruments, data analyses, and writing of the manuscript. KL participated in the data analyses and revisions of the manuscript. KWR and HJ participated in revising the manuscript.

Funding SWAN had grant funding from the National Institute on Aging (NIA), the National Institute of Nursing Research (NINR), and the NIH Office of Research on Women's Health (ORWH) (see Acknowledgements). Pink SWAN has grant support from the National Cancer Institute (CA 199137).

Data availability Public use datasets for SWAN data are available at the AgingResearchBiobank https://agingresearchbiobank.nia.nih.gov/studi es/swan/. The breast cancer data underlying this article were obtained through a separate source of funding (1 R01 CA199137-01) and are not shared publicly because of confidentiality. The data will be shared on reasonable request to the SWAN Coordinating Center, with review by the PI of that funding, Dr. Nancy Avis, and will require a formal data 
use agreement between the applicant's institution and the University of Pittsburgh.

Code availability Code for the analyses for this paper will be shared on reasonable request to the SWAN Coordinating Center and will require a formal use agreement between the applicant's institution and the University of Pittsburgh.

\section{Declarations}

Conflict of interest None, not applicable.

Ethical approval The institutional review boards at each sites approved the study protocol.

Consent to participate All sites obtained written informed consent from all of their study participants.

Consent for publication All authors made substantial contributions to the conception or design of the work; the acquisition, analysis, or interpretation of data; or the creation of new software used in the work; drafted the work or revised it critically for important intellectual content; approved the version to be published; and agreed to be accountable for all aspects of the work in ensuring that questions related to the accuracy or integrity of any part of the work are appropriately investigated and resolved.

Open Access This article is licensed under a Creative Commons Attribution 4.0 International License, which permits use, sharing, adaptation, distribution and reproduction in any medium or format, as long as you give appropriate credit to the original author(s) and the source, provide a link to the Creative Commons licence, and indicate if changes were made. The images or other third party material in this article are included in the article's Creative Commons licence, unless indicated otherwise in a credit line to the material. If material is not included in the article's Creative Commons licence and your intended use is not permitted by statutory regulation or exceeds the permitted use, you will need to obtain permission directly from the copyright holder. To view a copy of this licence, visit http://creativecommons.org/licenses/by/4.0/.

\section{References}

1. Gold EB, Sternfeld B, Kelsey JL, Brown C, Mouton C, Reame N, Salamone L, Stellato R (2000) Relation of demographic and lifestyle factors to symptoms in a multi-racial/ethnic population of women 40-55 years of age. Am J Epidemiol 152(5):463-473

2. Gold EB, Colvin A, Avis N, Bromberger J, Greendale GA, Powell L, Sternfeld B, Matthews K (2006) Longitudinal analysis of the association between vasomotor symptoms and race/ethnicity across the menopausal transition: study of women's health across the nation. Am J Public Health 96(7):1226-1235. https://doi.org/ 10.2105/AJPH.2005.066936

3. Davis SR, Panjari M, Robinson PJ, Fradkin P, Bell RJ (2014) Menopausal symptoms in breast cancer survivors nearly 6 years after diagnosis. Menopause 21:1075-1081. https://doi.org/10. 1097/GME.0000000000000219

4. Marino JL, Saunders CM, Emery LI, Green H, Doherty DA, Hicky M (2014) Nature and severity of menopausal symptoms and their impact on quality of life and sexual function in cancer survivors compared with women without a cancer history. Menopause 21:267-274. https://doi.org/10.1097/GME.0b013e3182976f46
5. Seib C, Porter-Steele J, McGuire A, McCarthy A, Balaam S, Anderson DJ (2017) Menopausal symptom clusters and their correlates in women with and without a history of breast cancer: a pooled data analysis from the women's wellness research program. Menopause 24:624-634. https://doi.org/10.1097/GME. 0000000000000810

6. Olin JL, St. Pierre M (2014) Aromatase inhibitors in breast cancer prevention. Ann Pharmacother 48:1605-1610. https://doi.org/10. 1177/1060028014548416

7. Maunsell E, Goss PE, Chlebowski RT et al (2014) Quality of life in MAP.3 (mammary prevention 3): a randomized, placebocontrolled trial evaluating exemestane for prevention of breast cancer. J Clin Oncol 32(14):1427-1436. https://doi.org/10.1200/ JCO.2013.51.2483

8. Yeo W, Pang E, Liem GS, Suen JJS, Ng RYW, Yip CCH, Li L, Yip CHW, Mo FKF (2020) Menopausal symptoms in relationship to breast cancer-specific quality of life after adjuvant cytotoxic treatment in young breast cancer survivors. Health Qual Life Outcomes 18:24-32. https://doi.org/10.1186/s12955-020-1283-x

9. Vincent AJ, Ranasinha S, Sayakhot P, Mansfield D, Teede HJ (2014) Sleep difficulty mediates effects of vasomotor symptoms on mood in younger breast cancer survivors. Climacteric 17:598604. https://doi.org/10.3109/13697137.2014.900745

10. Crandall C, Petersen L, Ganz PA, Greendale GA (2004) Association of breast cancer and its therapy with menopause-related symptoms. Menopause 11:519-530. https://doi.org/10.10987/ 01GME.000011706.40493.AB

11. Gupta P, Sturdee DW, Palin SL, Majumder K, Fear R, Marshall T, Paterson I (2006) Menopausal symptoms in women treated for breast cancer: the prevalence and severity of symptoms and their perceived effects on quality of life. Climacteric 9(1):49-58. https://doi.org/10.1080/13697130500487224

12. Avis NE, Levine B, Naughton MJ, Case DL, Naftalis E, Van Zee KJ (2012) Explaining age-related differences in depression following breast cancer diagnosis and treatment. Breast Cancer Res Treat 136(2):581-591. https://doi.org/10.1007/s10549-012-2277-0

13. Barba M, Pizzuti L, Sergi D et al (2014) Hot flushes in women with breast cancer: state of the art and future perspectives. Expert Rev Anticancer Ther 14(2):185-198. https://doi.org/10.1586/ 14737140.2013.856271

14. Meggetto O, Maunsell E, Chlebowski R, Goss P, Tu D, Richardson H (2017) Factors associated with early discontinuation of study treatment in the mammary prevention. 3 breast cancer chemoprevention trial. J Clin Oncol 35:629-635. https://doi.org/ 10.1200/HCO.2016.68.8895

15. Stan D, Loprinzi CL, Ruddy KJ (2013) Breast cancer survivorship issues. Hematol Oncol Clin North Am 27(4):805-27. https://doi. org/10.1016/j.hoc.2013.05.005

16. Fontein DB, Seynaeve C, Hadji P et al (2013) Specific adverse events predict survival benefit in patients treated with tamoxifen or aromatase inhibitors: an international tamoxifen exemestane adjuvant multinational trial analysis. J Clin Oncol 31(18):22572264. https://doi.org/10.1200/JCO.2012.45.3068

17. Gold EB, Pierce JP, Natarajan L et al (2009) Dietary pattern influences breast cancer prognosis in women without hot flashes: the Women's Healthy Eating and Living trial. J Clin Oncol 27(3):352-359. https://doi.org/10.1200/JCO.2008.16.1067

18. Huober J, Cole BF, Rabaglio M et al (2014) Symptoms of endocrine treatment and outcome in the BIG 1-98 study. Breast Cancer Res Treat 143(1):159-169. https://doi.org/10.1007/ s10549-013-2792-7

19. Liedke PE, Tu D, Shepherd L, Chavarri-Guerra Y, Pritchard K, Stearns V, Goss PE (2016) New onset vasomotor symptoms but not musculoskeletal symptoms associate with clinical outcomes on extended adjuvant letrozole-analyses from NCIC CTG 
MA.17. Breast 27:99-104. https://doi.org/10.1016/j.breast.2016. 02.010

20. van den Berg MJ, Mishra GD, van der Schouw YT, Herber-Gast G-CM (2014) Vasomotor menopausal symptoms are not associated with incidence of breast cancer in a population-based cohort of mid-aged women. Eur J Cancer 50:824-830. https://doi.org/10. 1016/j.ejca.2013.11.033

21. Reeves KW, Pennell M, Foraker RE, Crandall CJ, Stefanick M, Paskett ED (2018) Predictors of vasomotor symptoms among breast cancer survivors. J Cancer Surviv 12(3):379-387. https:// doi.org/10.1007/s11764-018-0677-9

22. Hart V, Sturgeon SR, Reich N, Sievert LL, Crawford SL, Gold EB, Avis NE, Reeves KW (2016) Menopausal vasomotor symptoms and incident breast cancer risk in the Study of Women's Health Across the Nation. Cancer Causes Control 27:1333-1340. https:// doi.org/10.1007/s10552-016-0811-9

23. Sowers MF, Crawford S, Sternfeld B et al (2000) Design, survey sampling and recruitment methods of SWAN: a multi-center, multi-ethnic, community-based cohort study of women and the menopausal transition. In: Lobos R, Marcus R, Kelsey JL (eds) Menopause. Academic Press, New York, pp 175-188

24. Neurgarten BL, Kraines RJ (1965) Menopausal symptoms in women of various ages. Psychosom Med 27:266-273

25. Avis NE, McKinlay SM (1991) A longitudinal analysis of women's attitudes toward the menopause: results from the Massachusetts Women's Health Study. Maturitas 13:65-79

26. Matthews KA, Wing RR, Kuller LH, Meilahn EN, Plantinga P (1994) Influence of the perimenopause on cardiovascular risk factors and symptoms of middle-aged healthy women. Arch Inter Med 154:2349-2355

27. Barsky AJ, Goodson JD, Lane RS, Cleary PD (1988) The amplification of somatic symptoms. Psychosom Med 50:510-519

28. Ferris BG (1978) Epidemiology standardization project (American thoracic society). Am Rev Respir Dis 118:1-120

29. Coghlin J, Hammond SK, Gann PH (1989) Development of epidemiologic tools for measuring environmental tobacco smoke exposure. Am J Epidemiol 130(4):696-704
30. Bromberger JT, Kravitz HM, Chang Y, Randolph JF Jr, Avis NE, Gold EB, Matthews KA (2013) Does risk for anxiety increase during the menopausal transition? Study of Women's Health Across the Nation. Menopause 20(5):488-495. https://doi.org/10.1097/ GME.0b013e3182730599

31. Radloff LS (1977) The CES-D scale: a self-report depression scale for research in the general population. Applied psychological measurement. West Publishing Co., Eagan, pp 385-401

32. Molenberghs G, Verbeke G (2005) Models for discrete longitudinal data. Springer Science+Business Media Inc, New York

33. Cleveland WS, Devlin SJ (1988) Locally weighted regression: an approach to regression analysis by local fitting. J Am Stat Assoc 83:596-610

34. Allison P (1995) Survival analysis using SAS: a practical guide. SAS Institute Inc, Cary, North Carolina

35. Chlebowski R, Mortimer J, Crandall C et al (2019) Persistent vasomotor symptoms and breast cancer in the Women's Health Initiative. Menopause 26(6):578-587

36. Dossus L, Jimenez-Corona A, Romieu I, et al (2014) C-reactive protein and postmenopausal breast cancer risk: results from the E3 N cohort study. Cancer Causes Control 25:533-539. https:// doi.org/10.1007/s10552-014-0355-9

37. Thurston RC, El Khoudary SR, Sutton-Tyrrell K et al (2011) Are vasomotor symptoms associated with alterations in hemostatic and inflammatory markers? Findings from the Study of Women's Health Across the Nation. Menopause 18:1-8

Publisher's Note Springer Nature remains neutral with regard to jurisdictional claims in published maps and institutional affiliations. 